LAWRENCE LIVERMORE N A T IO N A L LABORATORY

\section{Analysis of the time-reversal operator for planar dipole arrays}

D. H. Chambers, J. G. Berryman

January 27, 2004

IEEE International Symposium on Antennas and Propagation Monterey, CA, United States June 20, 2004 through June 26, 2004 
This document was prepared as an account of work sponsored by an agency of the United States Government. Neither the United States Government nor the University of California nor any of their employees, makes any warranty, express or implied, or assumes any legal liability or responsibility for the accuracy, completeness, or usefulness of any information, apparatus, product, or process disclosed, or represents that its use would not infringe privately owned rights. Reference herein to any specific commercial product, process, or service by trade name, trademark, manufacturer, or otherwise, does not necessarily constitute or imply its endorsement, recommendation, or favoring by the United States Government or the University of California. The views and opinions of authors expressed herein do not necessarily state or reflect those of the United States Government or the University of California, and shall not be used for advertising or product endorsement purposes. 


\title{
Analysis of the time-reversal operator for planar dipole arrays
}

\author{
D. H. Chambers* and J. G. Berryman \\ Lawrence Livermore National Laboratory \\ Livermore, CA 94551-9900
}

\section{Introduction}

The problem of imaging of targets in random media or cluttered environments is found in a wide variety of different applications, including ocean acoustics, medical ultrasound, geophysics, and radar. The solution often requires separating targets of interest from other scatterers, and compensating for wave speed variations in the medium. The problem is not usually the lack of data, but too much data, specifically the lack of a useful organizing principle for the data. The difficult part is separating the meaningful data from the remainder. It would therefore be most helpful if there were some means for skipping over those parts of the data that we do not really want to image very much, and looking at those parts (targets) that do interest us. This sounds challenging (maybe even impossible), but recent developments in acoustics make it clear that certain very limited imaging goals are achievable with much smaller data sets than are traditionally needed in, for example, seismic array processing [1]. Early versions of this new method have been given the names of "time-reversal acoustics" or "time-reversal mirrors," and have been developed most extensively by the French ultrasonics group led by Fink ([2], [3]])

Although these new ideas about target localization and imaging first arose in acoustics applications, the basic ideas are all wave-based and, therefore, pertinent with only a few changes to electromagnetic imaging modalities as well. Empirical approaches to time-reversal use specialized arrays that can both transmit and record arbitrary waveforms. These are available in acoustics but are difficult to build for typical radar frequencies. However, for imaging of targets, another approach using the same fundamental ideas as those behind the methods of empirical time-reversal is cheaper (because it requires less specialized hardware) and more effective. Instead of time-reversing and iterating the signals in the scattering process, we can map out once and for all the full response (or multistatic data) matrix. This matrix is the set of impulse responses for each combination of transmit and receive elements of the array. By Fourier transforming the elements of this temporal matrix, we obtain a frequency indexed set of complex but symmetric matrices. For each of these matrices we can perform the well-known singular value decomposition (SVD). Thus, we find all the desired information (all the singular vectors and singular values) simultaneously for each frequency. The singular vectors are the eigenvectors of the time-reversal operator (TRO) and the singular values are the square roots of the TRO eigenvalues. These can be used to estimate target location, reject clutter, or classify targets.

The response matrix method can be used when it is impractical to record the entire time-trace of the received signals. There are, however, two important issues especially pertinent to EM applications that need to be investigated. First, in acoustics the signals are pressure waves and do not carry polarization information, whereas in electromagnetics the signals are always transversely polarized, and can therefore carry information about either of two states of polarization. So there should be some additional information present in fully polarimetric radar data that is not available in acoustic data. Second, time-reversal imaging has been applied mostly to relatively small objects, often treated as point scatterers. Chambers and Gautesen [4] found that individual scatterers can have multiple eigenvalues and eigenvectors associated with them, when more than one of the physical properties of the objects show contrast with those of the surroundings (for example, bulk modulus and density for acoustics applications). This feature may be exploited for target discrimination and identification.

Recently, Tortel, Micolau, and Saillard [5] analyzed the electromagnetic time-reversal operator for sources and receivers in a ring surrounding a number of dielectric and conducting spheres and cylinders. We analyze the TRO for a planar array and show that for point scatterers, there can be up to six eigenvalues and eigenvectors of the time-reversal operator associated with each scatterer. These are associated with various configurations of the induced dipole moments in the scatterer, and depend not only on the geometry but also the polarization capability of the array.

\section{Derivation and decomposition of the multistatic data matrix}

Consider an array of $N$ short, crossed-dipole elements lying in the plane $z=-z_{a}$ (see Fig. 1 ), where $z_{a}$ is the distance between the plane and the sphere. The position of the $n t h$ element is given by the vector $\mathbf{r}_{n}=\left(\xi_{n}, \eta_{n},-z_{a}\right)$. 


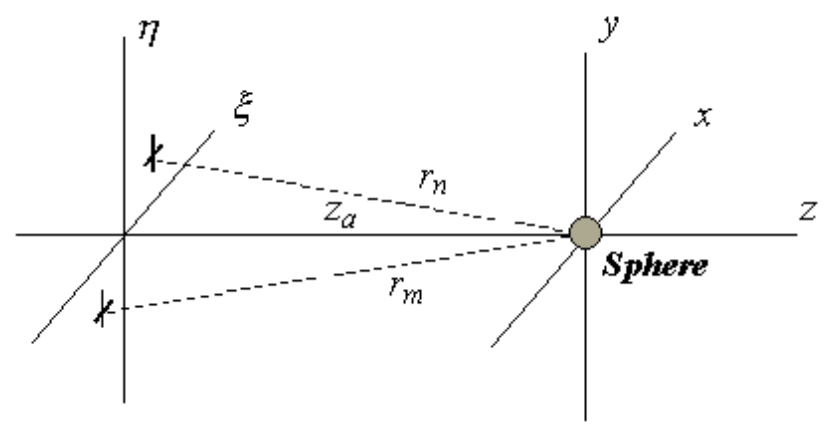

Fig. 1. Geometry of the array and sphere. Crossed dipole elements $m$ and $n$ are shown at distances $r_{m}$ and $r_{n}$, respectively, from the sphere. Distance from sphere to plane of the array is $z_{a}$.

Following Krauss [6], the electric field at the field point $\mathbf{r}$ radiated from the $n t h$ element is given by

$$
\begin{aligned}
\mathbf{E}_{n}^{(i)}\left(\mathbf{R}_{n}\right)= & \frac{i k e^{i k R_{n}}}{4 \pi \varepsilon_{0} c R_{n}} \hat{\mathbf{R}}_{\mathbf{n}} \\
& \times\left[\hat{\mathbf{R}}_{\mathbf{n}} \times\left(d_{H} I_{n}^{H} \hat{\mathbf{e}}_{\mathbf{x}}+d_{V} I_{n}^{V} \hat{\mathbf{e}}_{\mathbf{y}}\right)\right],
\end{aligned}
$$

where $c$ is the speed of light, $k$ is the wave vector, $\varepsilon_{0}$ is the electrical permittivity, and $\mathbf{R}_{n}=\mathbf{r}-\mathbf{r}_{n}$. The scalar $R_{n}$ is the magnitude of the vector $\mathbf{R}_{n}$ and $\hat{\mathbf{R}}_{\mathbf{n}}$ is the unit vector in the direction of $\mathbf{R}_{n}$. The horizontal and vertical dipoles in the element (lengths $d_{H}$ and $d_{V}$ ) are driven by the currents $I_{n}^{H}$ and $I_{n}^{V}$ respectively. The horizontal dipole is oriented parallel to the $x$ axis (unit vector $\hat{\mathbf{e}}_{\mathbf{x}}$ ) and the vertical dipole is oriented parallel to the $y$ axis (unit vector $\left.\hat{\mathbf{e}}_{\mathbf{y}}\right)$.

Let a sphere of radius $a \ll z_{a}$ be placed in front of the array, centered at the origin. The field incident on the sphere from the $n t h$ element can be approximated as a plane wave coming from the direction of the element. For a sphere much smaller than a wavelength, the field scattered from an incident plane wave is given to leading order $\left(\mathcal{O}\left((k a)^{3}\right)\right)$ [7] by

$$
\mathbf{E}^{(s)}(\mathbf{r})=-\frac{k^{2} e^{i k r}}{r}[\hat{\mathbf{r}} \times(\mathbf{m}+\hat{\mathbf{r}} \times \mathbf{p})],
$$

where $\mathbf{p}$ is the induced electric dipole moment and $\mathbf{m}$ is the induced magnetic dipole moment generated by the incident field. The moments are related to the incident field $\mathbf{E}_{n}^{(i)}$ evaluated at the position of the sphere $\mathbf{r}=0\left(\mathbf{R}_{n}=-\mathbf{r}_{n}\right)$ :

$$
\mathbf{m}=-m_{0} \hat{\mathbf{r}}_{n} \times \mathbf{E}_{n}^{(i)}\left(-\mathbf{r}_{n}\right), \quad \mathbf{p}=p_{0} \mathbf{E}_{n}^{(i)}\left(-\mathbf{r}_{n}\right),
$$

where $p_{0}=a^{3}\left(\tilde{n}^{2}-1\right) /\left(\tilde{n}^{2}+2\right), \tilde{n}^{2}=\varepsilon+i 4 \pi \sigma / \omega$, and $m_{0}=-i B_{1}^{m} / k^{3}$ (see [7]). The various factors are $\varepsilon$ the relative permitivity of the sphere, $\sigma$ the conductivity, $\omega$ the frequency, and $B_{1}^{m}$ is a quantity defined in reference [7] that determines the strength of the magnetic moment. When the conductivity of the sphere is small, $B_{1}^{m}$ can be neglected to leading order, so then the magnetic moment $\mathbf{m}$ does not contribute to the scattered field. In general, $p_{0}$ and $m_{0}$ are complex and can be represented in terms of magnitude and phase: $p_{0}=\left|p_{0}\right| e^{i \theta_{p}}, m_{0}=\left|m_{0}\right| e^{i \theta_{m}}$.

The scattered field induces voltages on each dipole of the array elements. From reference [8], the voltages induced on the dipoles of the $m t h$ element can be expressed as

$$
\begin{aligned}
V_{m}^{H} & =-d_{H}\left[\hat{\mathbf{r}}_{m} \times\left(\hat{\mathbf{r}}_{m} \times \hat{\mathbf{e}}_{\mathbf{x}}\right)\right] \cdot \mathbf{E}^{(s)}\left(\mathbf{r}_{m}\right), \\
V_{m}^{V} & =-d_{V}\left[\hat{\mathbf{r}}_{m} \times\left(\hat{\mathbf{r}}_{m} \times \hat{\mathbf{e}}_{\mathbf{y}}\right)\right] \cdot \mathbf{E}^{(s)}\left(\mathbf{r}_{m}\right) .
\end{aligned}
$$

Combining these with the previous expressions for the incident field (1) and scattered field (2) we can derive an expression for the coupling between the voltages in the $m t h$ receiving element and the currents in the $n t h$ transmitting element generated from the scattering by the sphere. Assembling the expressions for all $N$ elements gives a simple matrix expression relating the transmit currents to the receive voltages for all the dipole elements:

$$
\mathbf{V}=\mathbf{T} \mathbf{I}
$$

where $\mathbf{V}$ and $\mathbf{I}$ are vectors of length $2 N$ representing voltages and currents respectively, and $\mathbf{T}$ is a $2 N \times 2 N$ matrix representing the coupling. 


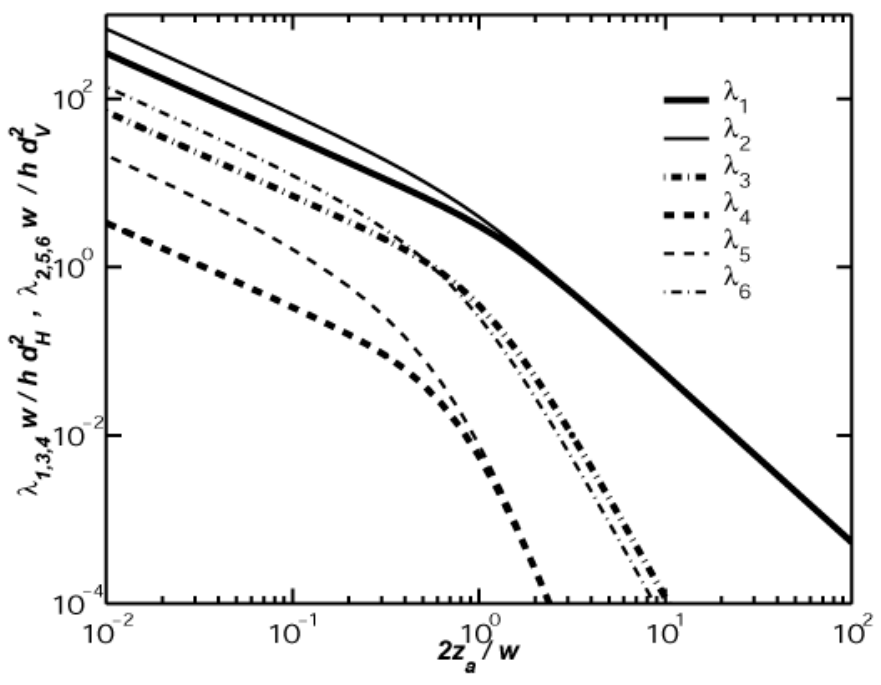

Fig. 2. Six singular values for a linear array and a conducting sphere as a function of normalized distance from the array, $2 z_{a} / w$. The singular values are scaled by $z_{a} / d_{H}^{2}$ for $\lambda_{1}, \lambda_{3}$, and $\lambda_{4}$ (heavy lines); and by $z_{a} / d_{V}^{2}$ for $\lambda_{2}, \lambda_{5}$, and $\lambda_{6}$ (light lines).

The eigenvalues and eigenvectors for the time-reversal operator are obtained by calculating the singular value decomposition of the $\mathbf{T}$ matrix:

$$
\mathbf{T} \Phi=\Lambda \Phi^{*},
$$

where the singular values $\Lambda$ are real and non-negative. The singular vectors $\boldsymbol{\Phi}$ are also the eigenvectors of the TRO, and the squares of the singular values are the eigenvalues of the TRO. A careful examination of the elements of the $\mathbf{T}$ matrix reveals that it can be expressed as a sum of six terms, each of which is an outer product of vectors:

$$
\begin{aligned}
\mathbf{T}= & -e^{i \theta_{p}}\left(\mathbf{g}_{1} \mathbf{g}_{1}^{T}+\mathbf{g}_{2} \mathbf{g}_{2}^{T}+\mathbf{g}_{3} \mathbf{g}_{3}^{T}\right) \\
& +e^{i \theta_{m}}\left(\mathbf{g}_{4} \mathbf{g}_{4}^{T}+\mathbf{g}_{5} \mathbf{g}_{5}^{T}+\mathbf{g}_{6} \mathbf{g}_{6}^{T}\right),
\end{aligned}
$$

where $\theta_{p}$ and $\theta_{m}$ are the phases of the induced electric and magnetic dipoles respectively. The first three terms are generated by the induced electric dipole, while the last three come from the induced magnetic dipole. The three terms for each dipole correspond to the three orthogonal orientations of the dipole moment in three dimensions. All terms contribute if the sphere is a good conductor, otherwise only the electric dipole terms are significant. This factorization means that rank of the $\mathbf{T}$ matrix (and the number of singular values) is no greater than six (three for nonconductive spheres).

Figure 2 shows the six singular values associated with a small conducting sphere as a function of range from a fully polarimatric linear array. These are two groups: one group associated with horizontal polarization $\left(\lambda_{1}, \lambda_{3}\right.$, and $\lambda_{4}$ ), and another with vertical polarization $\left(\lambda_{2}, \lambda_{5}\right.$, and $\left.\lambda_{6}\right)$. An array limited to only one polarization (horizontal or vertical) would generate only one of these groups. The three singular values in each group behave similarly in the near field $\left(2 z_{a} / w<1\right)$, staying within two decades of one another, but fall off at different rates in the far field $\left(2 z_{a} / w>1\right)$. Only the largest singular value for each polarization group $\left(\lambda_{1}\right.$ and $\left.\lambda_{2}\right)$ would likely be observed for spheres in the far field. Note that the largest singular values fall off at rates consistent with backscatter from a point target $\left(z_{a}^{-2}\right)$. Similar behavior has been calculated for dielectric spheres and for a circular planar array. In the latter case, the singular values are no longer separable into groups associated with horizontal and vertical polarization. Additional examples using both linear and circular arrays will be shown in the accompanying presentation.

\section{Conclusion}

We have derived an analytic expression for the time reveral operator (TRO) for the case of a planar array of crossed dipole elements illuminating a small sphere. The eigenvalues and eigenvectors of the TRO can be determined by performing a singular value decomposition (SVD) on the multistatic data matrix (response matrix) of the array. The eigenvalues of the TRO are the squares of the singular values, and the eigenvectors are identical to the singular vectors. We have shown that the maximum number of singular vectors associated with the sphere is equal to the number of orthogonal orientations of the dipole moments induced in the sphere when irradiated by the array. For a non-conducting sphere, only the electric dipole moment, with three possible orientations, contributes to the scattered field, so the number of possible singular values is three. For a conducting sphere, the induced magnetic dipole also 
contributes to the scattered field, so the number of possible singular values associated with the sphere is six, three for the electric dipole and three for the magnetic dipole. The actual number of singular values may be less, depending on the array configuration, polarization, and the location of the sphere. The number of orthogonal orientations of the dipole moments represent degrees of freedom in the scattering response of the sphere.

The singular values as a function of range were calculated for the examples of a linear array and a circular array when the sphere is positioned along an axis of symmetry of the array. In each case, the singular values behaved similarly when the sphere was in the near field of the array, but decayed at different rates for a sphere in the far field. Only the slowest decaying singular values would likely be observable for a sphere in the far field.

\section{Acknowledgments}

This work was performed under the auspices of the U. S. Department of Energy by the University of California, Lawrence Livermore National Laboratory under Contract No. W-7405-Eng-48. Research supported by DARPA.

\section{References}

[1] J. F. Claerbout, Fundamentals of Geophysical Data Processing with Applications to Petroleum Prospecting. New York: McGraw-Hill, 1976.

[2] C. Prada and M. Fink, "Eigenmodes of the time reversal operator: A solution to selective focusing in multiple-target media," Wave Motion, vol. 20, pp. 151-163, 1994.

[3] C. Prada, S. Manneville, D. Spoliansky, and M. Fink, "Decomposition of the time reversal operator: Detection and selective focusing on two scatterers," J. Acoust. Soc. Am., vol. 99, pp. 2067-2076, 1996.

[4] D. H. Chambers and A. K. Gautesen, "Time reversal for a single spherical scatterer," J. Acoust. Soc. Am., vol. 109, pp. 2616-2624, 2001.

[5] H. Tortel, G. Micolau, and M. Saillard, "Decomposition of the time reversal operator for electromagnetic scattering," J. Electromagn. Waves Appl., vol. 13, pp. 687-719, 1999.

6] J. D. Krauss, Antennas. San Francisco: McGraw Hill, 1988.

[7] M. Born and E. Wolf, Principles of Optics. New York: Pergamon Press, 1980.

[8] W. L. Stutzman and G. A. Thiele, Antenna Theory and Design. New York: Wiley, 1981. 